\title{
New insight on crystal and spot development in hard and extra-hard cheeses: Association of spots with incomplete aggregation of curd granules
}

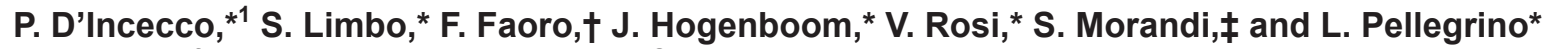 \\ *Department of Food Environmental and Nutritional Sciences, and \\ †Department of Agricultural and Environmental Sciences - Production, Landscape, Agroenergy, State University of Milan, 20133 Milan, Italy \\ łInstitute of Sciences of Food Production-Italian National Research Council, 20133 Milan, Italy
}

\begin{abstract}
Chemical composition and structure of different types of macroparticles (specks, spots) and microparticles (microcrystals) present in hard and extra-hard cheeses were investigated. Light microscopy revealed that the small hard specks had the structure of crystalline tyrosine, as confirmed by amino acid analysis. Spots showed a complex structure, including several curd granules, cavities, and microcrystals, and were delimited by a dense protein layer. Spots contained less moisture and ash than the adjacent cheese area, and more protein, including significantly higher contents of valine, methionine, isoleucine, leucine, tyrosine, and phenylalanine. Microcrystals were observed by light and electron microscopy and analyzed by confocal micro-Raman. Among others, calcium phosphate crystals appeared to consist of a central star-shaped structure immersed in a matrix of free fatty acids plus leucine and phenylalanine in free form or in small peptides. A hypothetical mechanism for the formation of these structures has been formulated.
\end{abstract}

Key words: hard and extra hard cheeses, cheese ultrastructure, calcium phosphate crystal, free amino acids, electron microscopy

\section{INTRODUCTION}

Hard and extra-hard are attributes used to define cheeses having a firm and brittle body texture (Codex Alimentarius, 1978). Hard and extra-hard cheeses share low moisture content, close structure, and a long ripening period. During ripening, many chemical, biochemical, and microbiological phenomena take place. The biochemical changes are very important for the de-

Received February 18, 2016.

Accepted April 14, 2016.

${ }^{1}$ Corresponding author: paolo.dincecco@unimi.it velopment of the flavor and texture of these cheeses and are characteristic of the different varieties. Proteolysis is the most relevant among the biochemical phenomena because of its complexity and final effect on the cheese taste. In fact, casein breakdown progressively produces large and medium peptides, followed by small peptides and free amino acids (FAA). Because FAA are rather stable, they tend to accumulate with the ripening time and may reach up to 20 to $24 \%$ on a cheese protein basis in 10- to 12-mo-old extra-hard cheeses (Masotti et al., 2010). Proteinases and peptidases that catalyze proteolysis in cheese originate from different sources, namely milk, rennet, and starter and nonstarter lactic acid bacteria (LAB). The LAB have complex enzyme patterns that release peptides and amino acids from the proteins into the cheese environment to satisfy their own nutritional requirements (Gatti et al., 2014). After vat processing, the loss of water, diffusion of salt, and formation of soluble molecules, such as FAA and lactate, are factors contributing to the increase of solute concentration and concomitant decrease of water activity in cheese throughout the whole ripening period. Beside these main events, minor changes, such as changes in water binding by new carboxylic and amino groups formed upon protein hydrolysis (McSweeney, 2004), contribute to lowering the water activity in cheese. The moisture content and water activity are strongly correlated in cheese throughout ripening (Marcos, 1993).

The increasing solute (salt, ions, FAA) concentration in the cheese water phase may give rise to aggregation and crystallization phenomena that result in the different types of structures in the interior and on the surface of different cheese varieties observed by some authors (Bianchi et al., 1974; Agarwal et al., 2006a; Tansman et al., 2015). Although the earliest studies date back to the 1900s (Babcock et al., 1903; Tuckey et al., 1938), a clear and unambiguous characterization of these structures has not yet been achieved. Moreover, a univocal association between the terminology (e.g., crystals, specks, dots, granules, spots, pearls) and the appearance of these structures is still lacking. 
In long-ripened extra-hard cheeses with a thick, dry rind, these structures develop inside the cheese and become visible when the wheel is cut. Typically, 2 different types of structures visible to the naked eye can be observed these structures are referred to as specks and spots in this article. Specks look bright white and firmer against the cheese matrix and are usually smaller than 2 to $3 \mathrm{~mm}$. Consumers (cheese lovers) appreciate the crispness of the specks when they chew them and their contribution to the overall cheese taste. Previous studies about specks reported that they contain clusters of tyrosine, cysteine, other FAA, calcium lactate, and magnesium (Shock et al. 1948) or tyrosine and phenylalanine (Giolitti and Mascherpa, 1970). More recently, Bottazzi et al. (1994) and Tansman et al. (2015) identified the specks in extra-hard cheeses as tyrosine crystals.

Spots are spherical and paler than the cheese and can be as large as 4 to $5 \mathrm{~mm}$. They appear to be amorphous and firmer with respect to the surrounding cheese matrix, and they become visible after 10 to 12 mo of ripening. Spots can become so numerous and conspicuous that they may influence the visual appeal of the cheese. Spots have been very little studied, and results have been inconsistent (Giolitti and Mascherpa, 1970; Bianchi et al., 1974; Tansman et al., 2015).

Besides specks and spots, extra-hard cheeses contain microscopic crystals, which have mostly been investigated in Cheddar cheese. However, some authors generically referred to crystals, without distinguishing between those that are microscopic and those that are visible to the naked eye (Bottazzi et al., 1982, 1994; Washam et al., 1985; Kaláb et al., 1987).

The aim of the present work was to shed light on the nature and origin of specks, spots, and microscopic crystals in extra-hard cheeses by using a multidisciplinary approach. Based on previous cheese structure studies (Ong et al., 2010; Schrader, 2012; D'Incecco et al., 2015), various microscopy techniques and different dyes (light and fluorescence, confocal, confocal micro-Raman, and transmission electron microscopy) were used in combination with chemical data to achieve an unambiguous characterization of these particles in cheese. Our ultimate goal was to formulate a hypothesis on the origin of these structures as they appear in hard and extra-hard cheeses. This knowledge will contribute useful information to understanding the bioavailability of selected minerals and nutrients in cheese. Furthermore, this knowledge could provide insights into the nature of these structures and might lead to new manufacturing strategies to control the formation of spots in commercial cheeses.

\section{MATERIALS AND METHODS}

\section{Cheese Samples and Collection of Specks and Spots}

Eleven extra-hard cheeses, ripened for 18 to $20 \mathrm{mo}$, were kindly provided by 2 dairies producing Grana Padano ( 7 cheeses) and Parmigiano-Reggiano (4 cheeses), respectively. Specks were harvested from the cheese using a pin, and spots were collected from the cheese with a spatula and then gently brushed to remove the cheese matrix on their surface. Specks and spots were collected separately from individual cheese samples in a sufficient amount (20-22 g) to conduct all the analyses. An equivalent amount of cheese was taken from the portion (0.5-cm thick) immediately surrounding the single spot, as shown in Figure 1, and used as a basis for reference. When necessary, a slice representative of the whole cheese was taken as well. Additional cheese portions were taken as required for microscopy investigations with various techniques. In particular, 20 spots of different size and taken from different cheeses were examined to determine their structural characteristics.

\section{Chemicals}

Glutaraldehyde, paraformaldehyde, cacodylate buffer, and osmium tetroxide were purchased from Agar Scientific (Stansted, UK). Toluidine blue, rhodamine, and single amino acids were purchased from Sigma Aldrich (Milan, Italy). Ninhydrin was purchased from Biochrom Ltd. (Cambridge, UK). Water purified with Milli-Q system (Millipore Corp., Bedford, MA) was used.

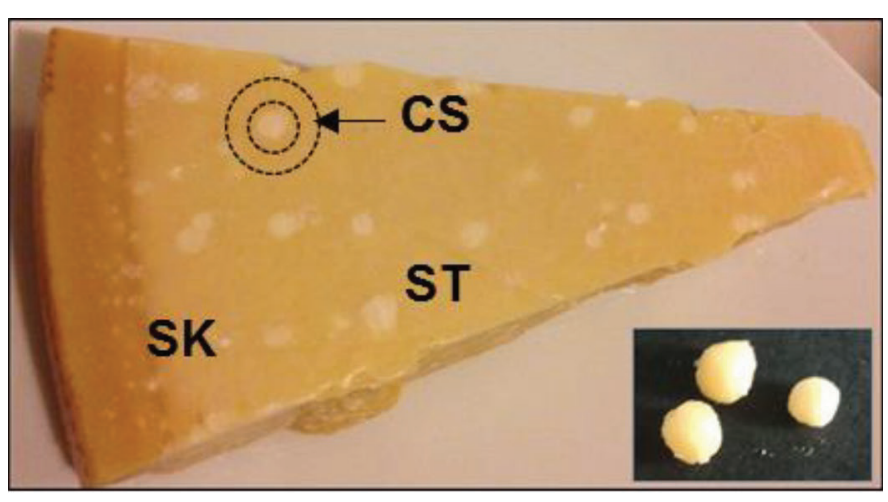

Figure 1. Photograph of extra-hard cheese showing specks (SK) and spots (ST). CS = cheese portion surrounding a spot (in sampling mode). Inset, isolated spots from the cheese. Color version available online. 


\section{Composition Analyses}

The ISO standard methods for cheese were used to determine the content of protein (method 27871; ISO, 2011), fat (method 1735; ISO, 2004a), ash (method 5545; ISO, 2008), and moisture (method 5534; ISO, 2004b), respectively. Content of calcium and phosphorus were determined by inductively coupled plasma MS spectrometer (Agilent Technologies, Milan, Italy).

The pattern of FAA was determined on the various cheese portions (including specks and spots) using the method described by Masotti et al. (2010). Briefly, the cheese portion was solubilized with sodium citrate, homogenized, and then deproteinized with sulfosalicylic acid. The extract was diluted (1:1) with lithium citrate buffer at $\mathrm{pH} 2.2$, filtered, and analyzed by ion exchange chromatography. The chromatographic separation was carried out on a Biochrom 30+ (Biochrom Ltd., Cambridge, UK) amino acid analyzer operated according to the manufacturer's instructions. The conditions involved an 8-step elution program with lithium citrate buffers of increasing $\mathrm{pH}$ and ionic strength, post-column derivatization with ninhydrin, and detection at 440 and $570 \mathrm{~nm}$. The quantification was carried out using 4-level calibration lines of the 21 amino acids in the range 0.75 to $22.5 \mathrm{mg} / \mathrm{L}$ and using norleucine (Sigma Aldrich) as an internal standard. Repeatability values of ISO standard 13903 (ISO, 2005) were fulfilled.

\section{Amino Acid Diffusion Trial}

To confirm the different diffusivity of individual FAA within the cheese, we injected $0.3 \mathrm{~mL}$ of an aqueous FAA solution at 3 times the concentration of the cheese water phase into the core of a spot-free cheese portion (a disk of $10 \mathrm{~g}$ ) using a microsyringe. The cheese portion was kept in a forced-ventilation thermostatic oven at $18^{\circ} \mathrm{C}$ for $18 \mathrm{~d}$ and then sampled as described in the Results. The FAA pattern and the moisture content were determined in each sampled portion.

\section{Light and Confocal Microscopy}

Specimens for light and fluorescence microscopy observations were thin sections obtained from resinembedded cheese samples prepared as described for transmission electron microscopy (TEM). Thin sections ( $4-5$ per sample) were dried directly on the microscope slide, stained, and subsequently washed. Two different stainings were performed: (1) toluidine blue ( $1 \%$ in water, wt/vol) for $5 \mathrm{~min}$ at room temperature to visualize the overall protein structure by light microscopy, and (2) rhodamine B $(0.5 \%$ in water, wt/ vol) for $5 \mathrm{~min}$ at room temperature. In the latter case, the sample was examined using a hg mercury lamp, with the following filters: excitation wavelength $=570$ $\mathrm{nm}$ and emission wavelength $=590 \mathrm{~nm}$. In addition, a 5 -cm cube of cheese was immersed in ninhydrin solution for $1 \mathrm{~h}$ and then cut with a blade until a thin section containing a spot was obtained. All samples were examined with an Olympus BX optical microscope (Tokyo, Japan) equipped with Nomarski interference contrast and QImaging Retiga camera (Surrey, BC, Canada). Specimens for confocal microscopy observations were cryo-sectioned with a CM1950 cryostat (Leica, Germany) and stained directly on the microscope slide with fast green $(0.1 \%$ in water, wt/vol). Sections were examined with a Nikon Vico video confocal microscope (Tokyo, Japan).

\section{Transmission Electron Microscopy}

Cubes of cheese (1-mm edge) were fixed in a mixture (wt/vol) of glutaraldehyde $3 \%$ and paraformaldehyde $2 \%$ in cacodylate buffer for $2 \mathrm{~h}$ at $4^{\circ} \mathrm{C}$, then washed with cacodylate buffer for $1 \mathrm{~h}$ and postfixed in osmium tetroxide ( $1 \%$ in water, wt/vol) for $2 \mathrm{~h}$. After dehydration in an ethanol series, the samples were embedded in London Resin White resin and cured at $60^{\circ} \mathrm{C}$ for $24 \mathrm{~h}$. Ultrathin sections (50-60 nm thick) were stained with uranyl acetate and lead citrate and examined with a Philips E208 transmission electron microscope (Aachen, Germany).

\section{Confocal Micro-Raman}

The Raman spectral data were collected in a range from 3,200 to $200 \mathrm{~cm}^{-1}$ Raman shift, using a confocal DXR Raman Microscope (Thermo Scientific, Waltham, MA). An Olympus $\times 50$ objective (numerical aperture $0.75)$ with a $50-\mu \mathrm{m}$ confocal pinhole was used to collect the Raman signal directly from a flat area of the sample (cut using a sharp knife) with a spatial resolution lower than $1 \mu \mathrm{m}$, without any preparation of the sample. A laser with an excitation wavelength of $780 \mathrm{~nm}$, lowenergy power $(5-10 \mathrm{~mW})$ to avoid overheating, and 400 lines/mm grating was used to record Raman spectra over the focalized area. A photobleaching time equal to 1 min was set up. For the specks, spectra were collected individually. For the microcrystals, a selected area was analyzed, and around 70 spectra were collected over the entire surface, using $10 \mu \mathrm{m}$ as the interval between positions. In particular, each sample was placed on an automated x,y mapping stage, and Raman spectra were obtained at different points of the selected surface, by moving it under the microscope objective. Autofocus at each map point was applied. Omnic Atlus software 


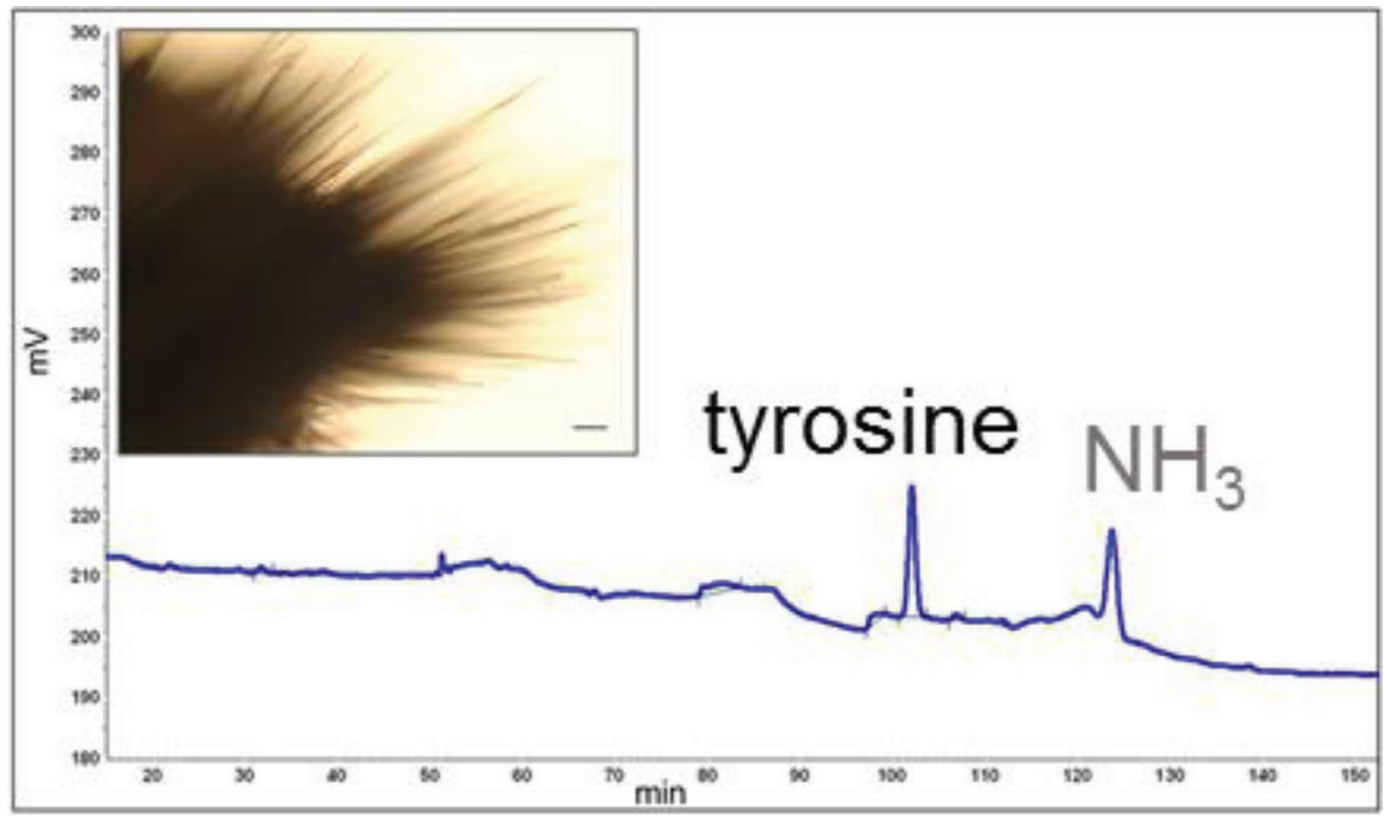

Figure 2. Free AA chromatography (absorbance at $440 \mathrm{~nm}$ as $\mathrm{mV}$ ) and (inset) light microscopy of a speck isolated from cheese. Bar $=200$ $\mu \mathrm{m}$. Color version available online.

(Thermo Fisher Scientific, Madison, WI) was used to obtain Raman spectra, perform spectrometer operations, and process data. All spectra were corrected for background contributions and an automated subtraction of cosmic ray peaks was employed.

\section{Statistical Analysis}

Statistical treatment of data was performed by means of SPSS Win 12.0 program (SPSS Inc., Chicago, IL). Data were analyzed by Student's $t$-test and one-way ANOVA. A $P<0.05$ was assumed as the significance limit.

\section{RESULTS}

\section{Speck Characterization}

Attempts to obtain a section of specks or to embed them in resin failed because they were too hard and packed. In contrast, specks in toto could be directly observed by light microscopy after isolation from cheese, and they showed the characteristic structure of crystalline tyrosine (Figure 2). The FAA pattern (Figure 2) indicated that specks were indeed tyrosine crystals of $>95 \%$ purity. The peak of ammonia in the chromatogram was derived from the elution buffers and thus was ignored in purity calculation. The Raman spectrum also confirmed the nature of the specks: the doublet
Raman bands at 828 and $848 \mathrm{~cm}^{-1}$ due to the Fermi resonance between ring fundamental and overtone were strongly evident, as was the ring-O stretching vibration located at 1,263 $\mathrm{cm}^{-1}$ (Culka et al., 2010). In addition, the highly resolved bands found in the fingerprint region $\left(1,614-250 \mathrm{~cm}^{-1}\right)$ were associated with the signals collected for the solid and pure (98-99\%) crystalline form of L-tyrosine (spectrum not shown).

\section{Spot Characterization}

Spot structure was first examined by light microscopy. A cheese portion was immersed in the protein-staining solution (ninhydrin) and then sectioned to reveal a spot inside that appeared to surrounded by a highly dense layer (Figure 3, arrowhead) that impaired stain permeation. However, some stain could permeate through preferential micro-pathways (junctions, indicated by arrows in Figure 3), making the structure visible. Thin sections of resin-embedded spots, stained with toluidine blue, gave more insight into the inner structure (Figure 4a). The spot appeared to be made of several curd particles having clean-cut irregular shapes and a size up to $0.5 \mathrm{~mm}$. The darker lines, corresponding to the junctions between curd particles, were richer in protein than the particle body. Several openings, including a large hole, where the junctions met, were visible in the section (Figure $4 \mathrm{~b}$ ). Although the cheese around the spot showed the same composite structure (not shown), 


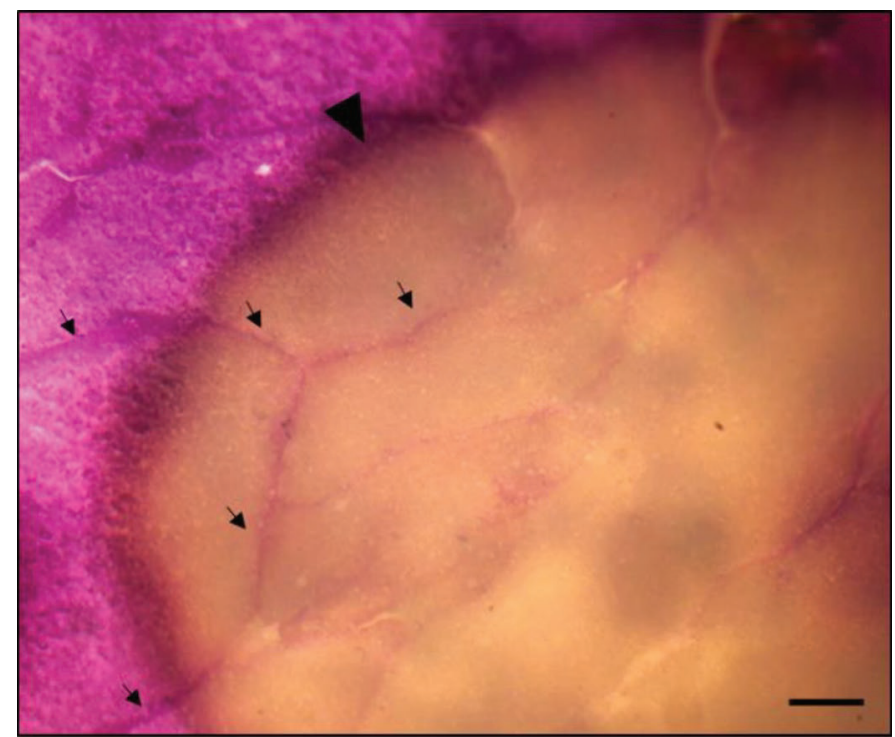

Figure 3. Handmade section of Grana Padano cheese including a spot: light microscopy after ninhydrin staining revealed a dense layer limiting the spot (arrowhead) and the junctions among curd granules (arrows). Bar $=200 \mu \mathrm{m}$. Color version available online.

large cavities were observed only inside spots. With respect to the curd particles, the junctions appeared as thick protein strings almost free of fat (Figure 4c) and rich in microcrystals (arrows). Microcrystals were also observed in the cheese matrix outside the spot, as further discussed.

The ultrastructure of the spot, examined by TEM, proved to be remarkably different from that of the surrounding cheese (Figure 5). In particular, the interface between protein (Figure 5, in gray) and fat (in white) was more irregular and fringed by crystal-like particles (Figure 5a) with respect to the cheese surrounding the spot (Figure 5b).

The chemical composition of the spots was compared with that of the adjacent cheese and of the whole cheese. Five different cheeses were individually analyzed (Table 1). Spots proved to be significantly richer in protein $(P$ $<0.00)$ and poorer in moisture $(P<0.00)$ and ash $(P$ $<0.00$ ) with respect to the surrounding cheese portion, which did not differ from the whole cheese. Fat content was not different $(P<0.17)$. The content of both calcium and phosphorus was $10 \%$ lower in the spot than in the cheese, with a $\mathrm{Ca}: \mathrm{P}$ molar ratio of 1 in both zones (not shown). The protein fraction was characterized by capillary zone electrophoresis as described in our previous work (Masotti et al., 2010). No difference could be shown in either casein or peptide patterns between the spots and the rest of the cheese (not shown), indicating that the primary proteolysis had proceeded to the

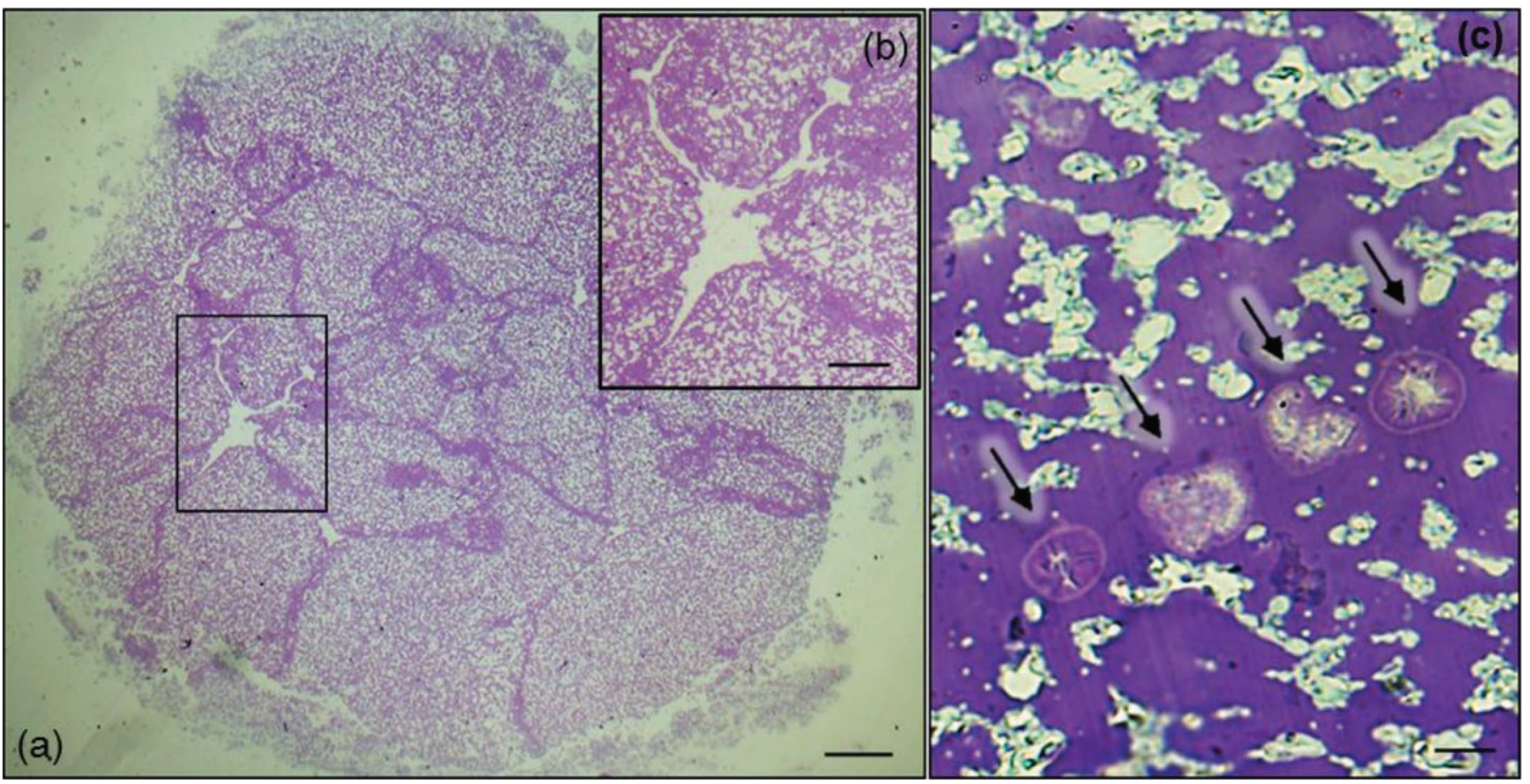

Figure 4. Light microscopy of spot semi-thin section $(2-5 \mu \mathrm{m})$ stained with toluidine blue. (a) The curd junctions are visible as darker lines. Bar $=200 \mu \mathrm{m}$. (b) Detail of the large hole in which junctions converge. Bar $=50 \mu \mathrm{m}$. (c) Microcrystals (arrows) along a curd junction. Bar $=10 \mu \mathrm{m}$. Color version available online. 

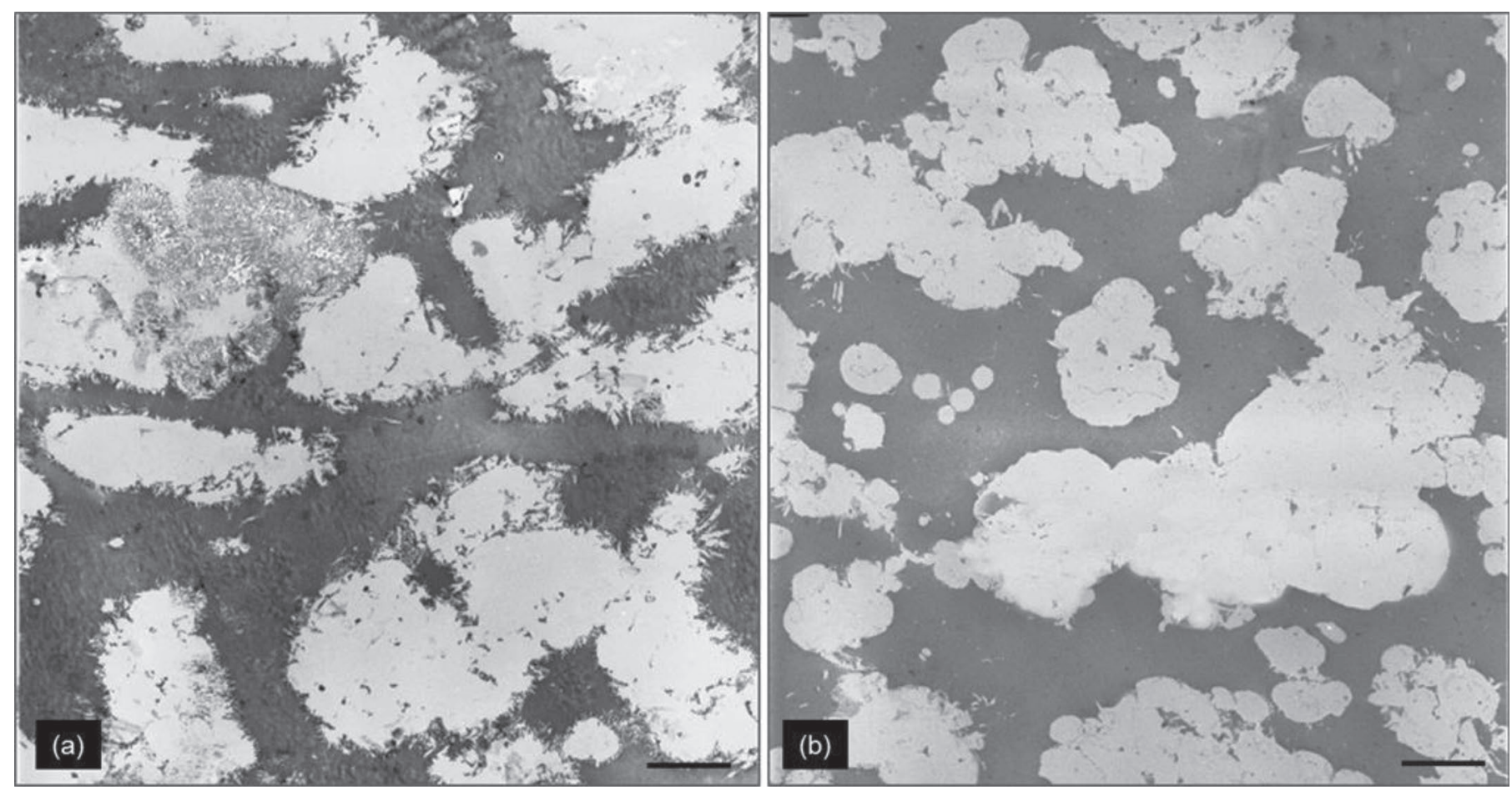

Figure 5. Ultrathin section of spot ultrastructure (a) in comparison with the surrounding cheese (b). The network of electron dense proteins shows a more irregular profile in the spot, particularly at the interface with the electron transparent fat matrix. Bar $=1 \mu \mathrm{m}$.

same extent within and outside the spot. In contrast, significant differences were found in the FAA patterns of the 2 zones.

To compare data of cheese portions (i.e., spots and surrounding cheese) having different moisture contents, and considering that FAA are soluble molecules, values were expressed on the respective moisture content. Furthermore, data from both Grana Padano and Parmigiano-Reggiano were pooled to increase statistical significance. In particular, spots contained significantly higher amounts (from 4 to 12 times) of 6 FAA: valine $(P<0.00)$, methionine $(P<0.00)$, isoleucine $(P<0.00)$, leucine $(P<0.00)$, tyrosine $(P<$ $0.00)$, and phenylalanine $(P<0.00)$ (Figure 6$)$. It is worthy of remark that the content of the other FAA was the same as in the cheese portion around the spot. Nevertheless, this last portion had the usual FAA pat- tern we observed for the 2 target cheeses in previous studies (Cattaneo et al., 2008; Masotti et al., 2010). To achieve direct confirmation of such a different composition in individual FAA within the cheese, a simple experiment was carried out. A water solution having the approximate concentration of FAA in the cheese water phase was injected into a spot-free cheese slice. After a resting time suitable to allow diffusion of the solution, the cheese was sampled taking 3 distinct portions: one circular, corresponding to the injection point (mimicking the spot), and 2 concentric rings around it, as shown in Figure 7 (inset). Despite the approximated experimental conditions and sampling procedure, the data confirmed that the same 6 FAA were retained in the zone where the mixture was injected, but the others diffused to the surrounding portions, largely reaching an equilibrium (Figure 7 ).

Table 1. Chemical composition (\%) of spot, cheese portion around it, and whole cheese ${ }^{1}$

\begin{tabular}{lcccc}
\hline Area & Moisture & Fat & Protein & Ash \\
\hline Spot & $24.2^{\mathrm{a}} \pm 1.2$ & $27.5^{\mathrm{a}} \pm 2.6$ & $37.6^{\mathrm{a}} \pm 1.3$ & $3.9^{\mathrm{a}} \pm 0.1$ \\
Cheese around the spot & $29.5^{\mathrm{b}} \pm 1.0$ & $29.6^{\mathrm{a}} \pm 1.2$ & $33.5^{\mathrm{b}} \pm 0.6$ & $4.4^{\mathrm{b}} \pm 0.3$ \\
Whole cheese & $29.8^{\mathrm{b}} \pm 1.0$ & $29.9^{\mathrm{a}} \pm 1.1$ & $33.5^{\mathrm{b}} \pm 0.2$ & $4.4^{\mathrm{b}} \pm 0.2$ \\
\hline
\end{tabular}

$\overline{\mathrm{a}, \mathrm{b}}$ Means in the same column with different letters are significantly different $(P=0.05)$.

${ }^{1}$ Data are means \pm SD based on duplicate analyses of 5 cheeses. 


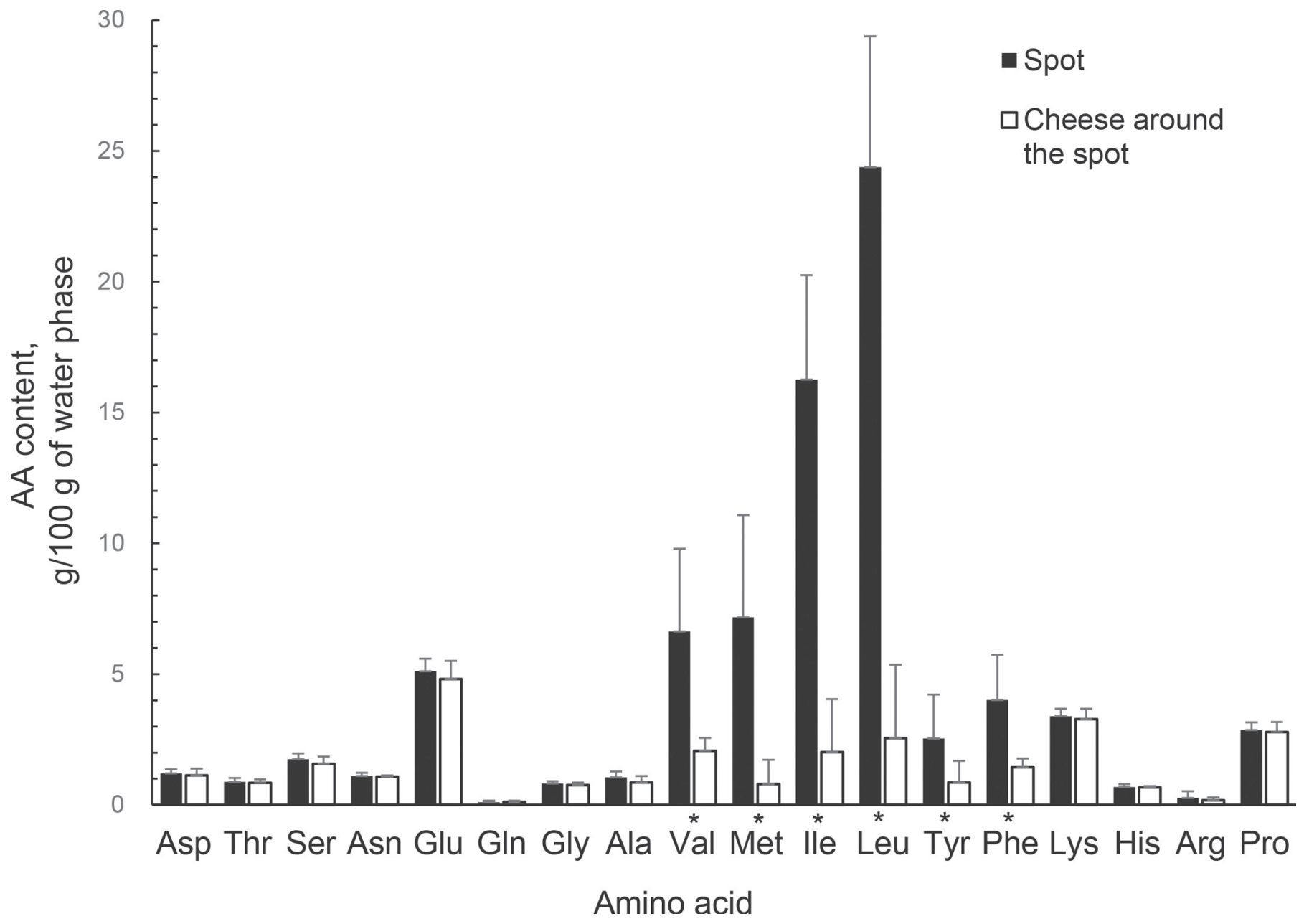

Figure 6. Free AA content of the spot and the cheese portion around it. Data are expressed as means (columns) of 11 cheeses \pm SD (bars). *Values are statistically different $(P<0.05)$.

\section{Microscopic Crystals}

Microscopic crystals were investigated through various microscopy techniques. Observations by fluorescence microscopy of cheese semi-thin sections, after resin embedding and staining with rhodamine $\mathrm{B}$, allowed seeing a huge number of microscopic crystals in bright red (Figure 8a). Confocal microscopy showed that crystals had different shapes (i.e., circular, oval, or kidney-shaped), and their cores were not fluorescent, indicating a different composition in respect to their peripheral zone (Figure 8b). The number of crystals ranged from 30 to 100 crystals $/ \mathrm{mm}^{2}$ in 18 - to 20 -mo ripened cheeses and did not noticeably differ between cheese and inside spots. Crystals with the same structure were detected in younger (3 and 6 mo of ripening) extra-hard cheeses, although in a lower amount (not shown).
Microscopic crystals observed by TEM showed a complex ultrastructure (Figure 8c). Three main zones could be outlined: (1) a central star-shaped crystal, (2) an intermediate zone, and (3) a peripheral compact shell at the interface with the protein matrix. Figure 8d shows both the external shell and the intermediate zone were constituted by fibrils with prismatic morphology, radially ordered and packed around the central crystal.

Confocal Raman microscopy, which does not require any sample preparation, was used to obtain information on the chemical composition. Area maps of the crystals, detected both inside and outside the spot, confirmed the presence of distinct regions, as observed by TEM. Spectra of the central star-shaped crystal matched the calcium phosphate spectrum, dominated by the very strong band at $986 \mathrm{~cm}^{-1}$ and the medium band at $878 \mathrm{~cm}^{-1}$ (Figure 9) that derived from the symmetric stretching mode of the phosphate group (Sauer et al., 


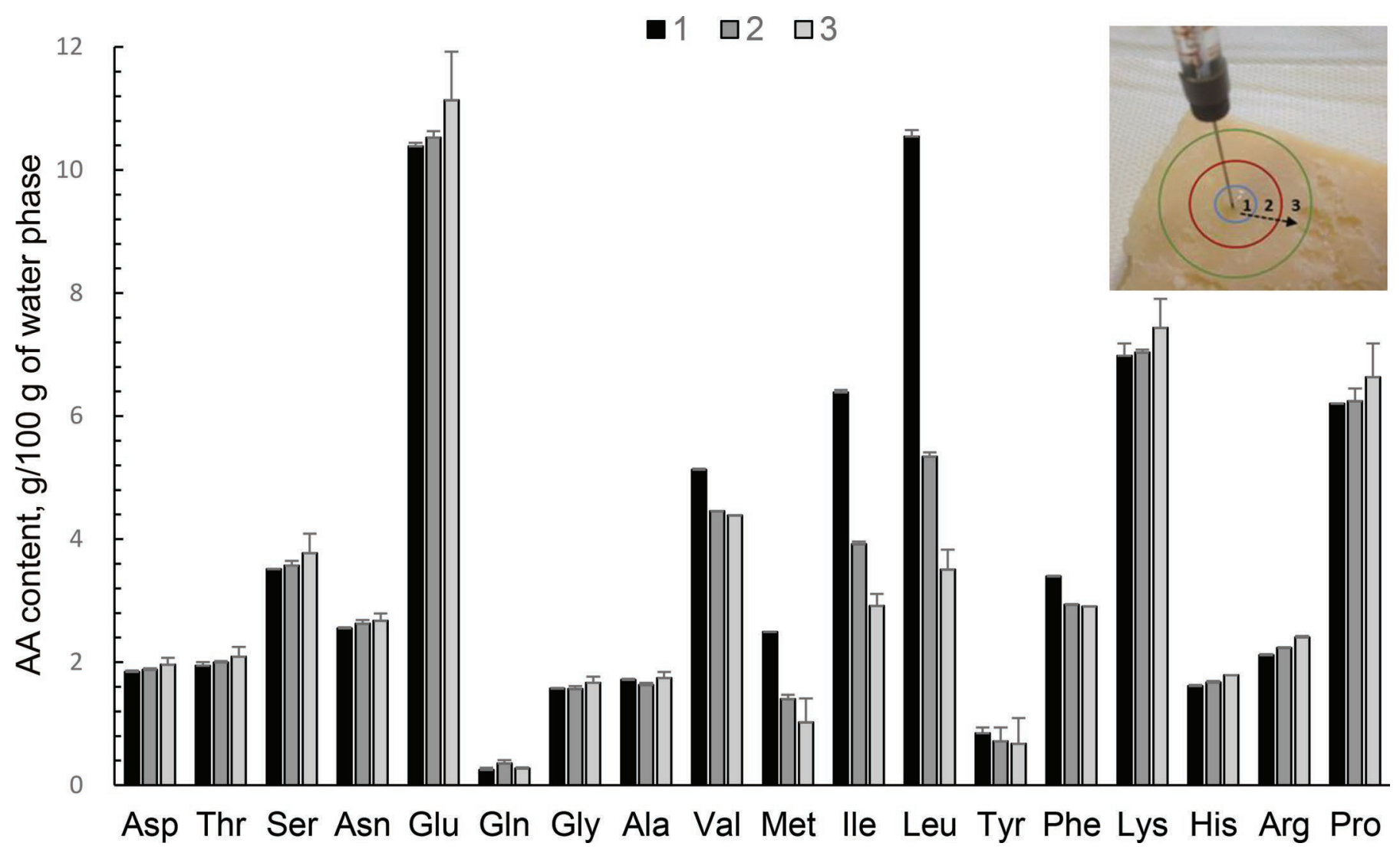

Figure 7. Free AA content of the portions 1 to 3 taken from cheese after the diffusion of $0.3 \mathrm{~mL}$ of aqueous AA solution injected in 1 (inset). Data are the means of duplicate analyses. Error bars indicate the SD. Color version available online.

1994). The band at $1,081 \mathrm{~cm}^{-1}$ corresponded to the stretching vibration $\left(\boldsymbol{v}_{3}\right)$ of $\mathrm{PO}_{4}{ }^{3-}$, and the band at 588 $\mathrm{cm}^{-1}$ corresponded to the P-O and O-P-O stretching and bending modes $\left(\boldsymbol{v}_{4}\right)$ of the same group. In calcium phosphate crystals, the minerals can be identified by the position and shape of the main bands. Raman shifts and assignment for some calcium phosphate minerals were studied by Koutsopoulos (2002). The SavitskyGolay second derivative of the spectra highlighted the presence of other weak bands attributable to the dibasic calcium phosphate dehydrate form. No calcium phosphate was detected in other parts of the complex crystal structure. In fact, in the outer zone, which appeared as a dark area in the optical magnification used for the Raman acquisition, spectra presented bands that arose from both free fatty acids and proteins (Figure 10a). The spatial distribution of fatty acid or protein with respect the calcium phosphate within the crystal area is shown in Figure 10b (in blue). It is evident that the crystal is immersed in the cheese matrix. A deeper analysis of the spectra of this portion provided a major characterization of the protein structure contribution. Usually, the amide I and III peaks in a protein are less sharply resolved in comparison with the signals of small peptides (Jenkins et al., 2005). In other words, Raman spectral signatures of single amino acids are retained in a peptide or protein, being largely derived from their side chain or backbone. The higher the resolution and intensity of the bands in a complex spectrum, the more probable the contribution of amino acids, small peptides, or both. Thus, in the original spectrum collected from the cheese matrix around the phosphate crystal, some specific bands of amino acids were evident. In particular, a spectral subtraction of an unsaturated free fatty acid spectrum (considered as a background) from the original spectrum of the external region returned the profile of leucine ( $>60 \%$ match with the pure spectrum of L-leucine) and phenylalanine (53\% match with the pure spectrum of L-phenylalanine). Leucine was mainly characterized by bands at $1,237 \mathrm{~cm}^{-1}$ due to the twisting of $\mathrm{CH}_{2}$ and by bands at 1,187 and $1,132 \mathrm{~cm}^{-1}$ due to the rocking of $\mathrm{NH}_{3}{ }^{+}$, and phenylalanine showed a very intense band around $1,000 \mathrm{~cm}^{-1}$. These results indicated that the protein component around the crystal was mainly due to these 2 amino acids, present in free form or in small peptides. 

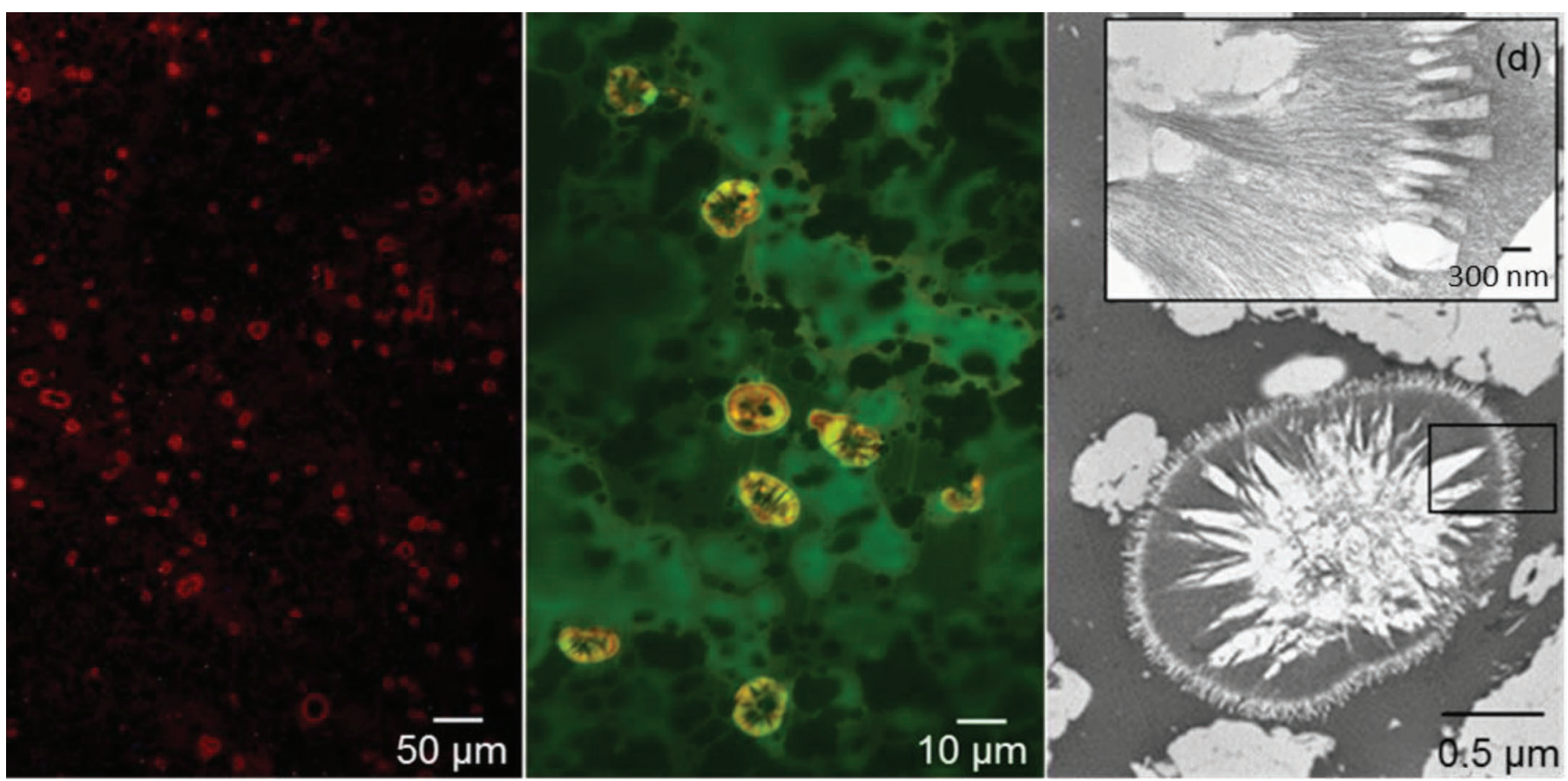

Figure 8. Calcium phosphate crystals in cheese. (a) Fluorescence microscopy of crystals stained with rhodamine B. (b) Video confocal microscopy of crystals stained with fast green and observed with tetramethylrhodamine isothiocyanate (TRITC) and fluorescein isothiocyanate (FITC) filters, revealing a nonfluorescent core. (c) Ultrathin section of a crystal showing a star shaped zone in the center; at the interface with protein matrix, the fibrillar structure of the crystal is visible (d). Color version available online.

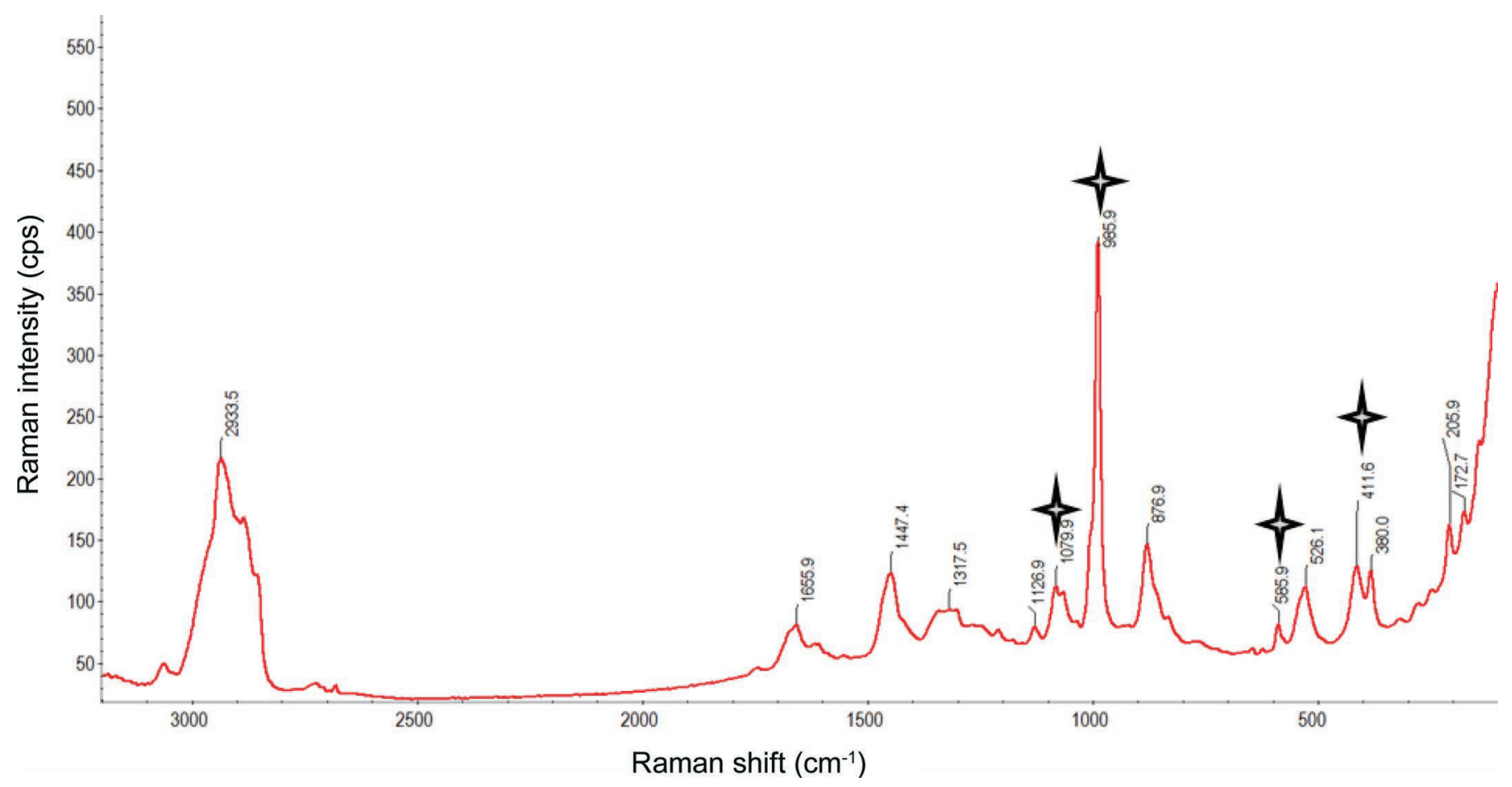

Figure 9. Raman spectrum of calcium phosphate crystal in cheese. Stars represent typical signals of the salt. Color version available online. 
Other crystalline structures were randomly detected within the cheese and analyzed by means of microRaman spectroscopy. Only calcium carbonate crystals were identified so far. They appeared spheroidal in shape and translucent: the peaks at 1,083, 1,410, 713, and $284 \mathrm{~cm}^{-1}$ confirmed their nature (Tlili et al., 2001; data not shown). The presence of calcium carbonate in cheese seems to be attributable to the microbial metabolism that produces $\mathrm{CO}_{2}$ (Gaucheron et al., 1999).

\section{DISCUSSION}

\section{Speck Characterization}

By evaluating the confocal micro-Raman spectrum and FAA composition of specks, we obtained a tyrosine purity $>95 \%$, confirming previous reports indicating the presence of this amino acid in these structures (Bottazzi et al., 1994; Tansman et al., 2015). Free tyrosine concentration increases in the whole cheese throughout the ripening process, as do all other FAA (Pellegrino et al., 1997). In our samples of Grana Padano and Parmigiano-Reggiano, free tyrosine concentration was approximately $0.8 \mathrm{~g} / 100 \mathrm{~g}$ of water phase (Figure 6); that is, about 10 times higher than its water solubility at room temperature (Grosse Daldrup et al., 2009). Therefore, formation of crystals spread within the cheese would suggest that progress of proteolysis is not homogeneous in the matrix, thus leading to FAA accumulation, including tyrosine, preferentially into micro-openings until saturation. Furthermore, we have obtained by light microscopy further structural details of tyrosine crystals from cheese (Figure 2), showing that the former reported description (Tansman et al., 2015) is due to a micro-spike of the crystalized amino acid. When observed by atomic force microscopy in protein hydrolysates (McPherson et al., 2012), tyrosine crystals appeared to be covered by a stable layer of 3-nm particles, likely represented by micellar arrangement of small peptides present in the medium and able to prevent resolubilization of crystals once formed. Given the remarkable content of peptides in ripened cheeses, this aspect would be worthy of investigation.

\section{Spot Characterization}

In contrast to the hard specks, spots were easily embedded in resin and cut into thin sections, which allowed a deeper characterization by various microscopy techniques. Previous studies on spots in cheese did not investigate their structure and ultrastructure (Giolitti and Mascherpa, 1970; Bianchi et al. 1974; Tansman et al., 2015). Unexpectedly, when the cheese was directly stained with ninhydrin and observed by light microscopy, the original grains of curd were still visible. During cheese manufacturing, curd grains $(20-50 \mathrm{~mm})$ obtained by cutting the rennet gel are left in hot whey for 50-60 min to settle and aggregate at the bottom of the vat. Further fusing and shrinking of curd grains are induced by the subsequent acidification of the cheese loaf when kept in mold for $48 \mathrm{~h}$. Grana Padano and Parmigiano-Reggiano loaves are not pressed, so high temperature and low $\mathrm{pH}(5.2-5.3)$ play a major role in promoting whey draining (Pellegrino et al., 1997) and the tight aggregation of curd grains. However, as already noted, these grains appeared as separate units even several months later. The junctions between contiguous grains are low in fat because many fat globules

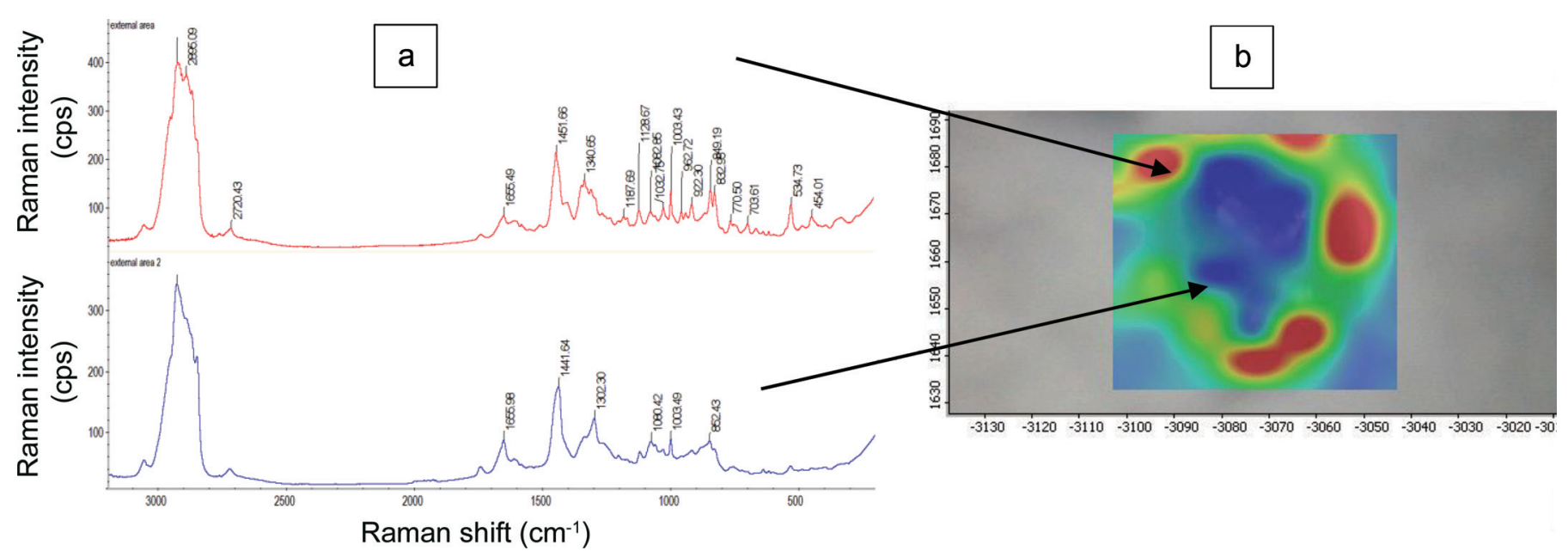

Figure 10. Raman spectra (a) and images (b) of spatial distribution of lipid and protein components [red and green areas (gray and light gray areas)] around the crystal [covered by the blue (black) area in the middle]. High, medium, and very low component concentrations are indicated as red, green, and blue, respectively. Color version available online. 
escape the protein network at the surface of the grains before they stick together. For the grains having either an irregular shape or a different size, their aggregation during settling forms an internal hole, connected by several openings and radial junctions. This pattern was frequently observed within the spots (Figure 3b). In the young cheese, even before the spot formation, these hollow cavities likely represent preferential zones where whey stagnates and entrapped bacterial cells find substrates for growth (Le Boucher et al., 2016). Growth of bacterial colonies as affected by local concentration of substrates in cheese is receiving increasing attention (Silva et al., 2013; Jeanson et al., 2015). Although no intact bacterial cells were detected by TEM in cavities within the spot or in the surrounding cheese, likely due to the prolonged ripening process, an indirect, preliminary confirmation of this hypothesis came from measuring the amount of total DNA extracted from the spot and the surrounding cheese following the procedure of Cremonesi et al. (2007). In fact, the amount of DNA was more than 3 times higher in the spots than in the surrounding cheese (72.94 vs. $22.41 \mu \mathrm{g} / \mathrm{mg}$ ).

Spots proved to be significantly drier with respect to the surrounding cheese portion and to contain more protein and less ash (Table 1), confirming the data reported by Tansman et al. (2015). In addition, we showed the cheese portion just around the spot had the same chemical composition as the rest of the cheese, confirming that the spot is a fully isolated unit. The dense layer we observed around it by light microscopy is likely explains this observation (Figure 3, arrowhead). Overall, these findings point to a migration of whey with consequent draining of solutes occurring locally within the cheese where a spot would originate later.

Spots were also reported to have a different FAA composition compared with the whole cheese. Bianchi et al. (1974) found spots in 18- and 25-mo ripened Grana Padano cheeses to contain (g/100 g) leucine (9.86), isoleucine (4.96), methionine (1.64), valine (1.52), glutamic acid (1.84), and asparagine (1.63) as dominant FAA. However, these authors did not find that the last 2 FAA were equally abundant in the whole cheese. Recently, Tansman et al. (2015) detected only 1\% of free leucine in spots from a 24-mo old Parmigiano-Reggiano cheese, but they did not provide an explanation for the discrepancy between this result and the literature. We obtained FAA data similar to those of Bianchi et al. (1974), but we interpret the information differently. The key to understanding the genesis of the spot relates to the chemical properties of the different FAA present in the spot and in the cheese regardless of their concentrations. In fact, the $6 \mathrm{FAA}$ we detected at much higher levels in the spot (i.e., leucine, isoleucine, methionine, valine, phenylalanine, and tyrosine; Figure 6) are all hydrophobic because of aromatic or branched chain structures, and thus they are insoluble in the water phase of cheese. This shared FAA characteristic leads us to hypothesize that local water movements are responsible for the different distribution of FAA and consequently for the spot blowing in hard and extrahard cheeses. This hypothesis was confirmed by our experiment mimicking the migration of FAA within the cheese.

As already mentioned, the cavities in which the residual whey stagnates in young cheese could be the sites where bacterial colonies develop. During cheese ripening, the water phase in these sites becomes more concentrated in solutes due to lactic acid bacteria metabolism and, at a later stage, to cell lysis. The water phase progressively migrates through the curd grain junctions, dragging solutes, including polar FAA in preference to the hydrophobic ones. Consequently, fewer water-soluble molecules concentrate in a restricted area that evolves into a spot from which water moves away radially (Figure 7), also pulling away salts. For this reason, many crystals were visible in the junctions (Figure 4c).

\section{Microscopic Crystal Characterization}

Microcrystals appeared to be spread throughout the whole cheese. Among these crystals, we identified calcium phosphate by micro-Raman directly on the cheese, without any sample preparation. The central star-shaped crystals observed by TEM (Figure 8c) were clearly assigned to selected types of calcium phosphate. Gaucheron et al. (1999) demonstrated that the supersaturation of calcium phosphate salts strongly increases during cheese ripening because of the rise in $\mathrm{pH}$, explaining the salt precipitation. Unfortunately, the few references that discuss the ultrastructure of crystals in cheese give no indication about the nature of the calcium phosphate. In our study, the micro-Raman analysis produced sufficient information about their morphological features and complex ultrastructure. In fact, this technique allowed gathering evidence for the first time that the fibrillary layer surrounding the central crystal contains leucine and, to a lower amount, phenylalanine. These hydrophobic amino acids are reported to limit growth of calcium phosphate crystals due to their absorption on the crystal surface, which blocks the active growth sites (Dalas et al., 2008). Although calcium lactate could form in cheese through lactic acid fermentation, the chelation of calcium by the phosphates should be predominant (Heertje et al., 1981), in agreement with the amount of calcium phosphate crystals we detected. Agarwal et al. (2006b) reported that calcium lactate crystals only form when $\mathrm{pH}$ is higher than 5.1. 
We could not find calcium lactate crystals, although we cannot exclude their presence in extra-hard cheeses because different unidentified crystalline structures were observed by TEM. Some researcher detected calcium lactate crystals in cheeses other than Grana Padano and Parmigiano-Reggiano (Washam et al., 1985; Tansman et al., 2014), whereas only Bottazzi et al. (1982) detected them in 14-mo ripened Grana cheese, indicating their low incidence (i.e., $2-3 / \mathrm{mm}^{2}$ ).

The presence of leucine crystals is likely within the spot where free leucine showed a concentration that was 10 times higher in the water phase than in the cheese (Figure 6). Crystalline leucine was also detected by Tansman et al. (2015) in pearls collected from Parmigiano-Reggiano cheese.

\section{CONCLUSIONS}

Combining background knowledge about extra-hard cheese manufacturing and ripening with new information about composition and structure of spots achieved in this study, we can hypothesize that spot development originates with an incomplete aggregation of curd granules and a consequent local whey stagnation. Growth and lysis of bacterial cells entrapped in these microcavities influence both metabolite availability and micro-environmental conditions, which in turn regulate diffusion and crystallization of solutes locally. Therefore, practices targeted at improving syneresis, such as fine-tuning the temperature control and curd grain size, would greatly reduce spot number and the occurrence of other confined phenomena.

\section{REFERENCES}

Agarwal, S., J. R. Powers, B. G. Swanson, S. Chen, and S. Clark. 2006a. Cheese $\mathrm{pH}$, protein concentration, and formation of calcium lactate crystals. J. Dairy Sci. 89:4144-4155.

Agarwal, S., K. Sharma, B. G. Swanson, G. U. Yüksel, and S. Clark. 2006b. Nonstarter lactic acid bacteria biofilms and calcium lactate crystals in Cheddar cheese. J. Dairy Sci. 89:1452-1466.

Babcock, S. M., L. H. Russel, A. Vivian, and U. S. Baer. 1903. Condition affecting the development of white specks in cold-curd cheese. Pages 180-183 in Wisc. Agric. Exp. Stn. 19th Annu. Rep. Forgotten Books, Madison, WI.

Bianchi, A., G. Beretta, G. Caserio, and G. Giolitti. 1974. Amino acid composition of granules and spots in Grana Padano cheeses. J. Dairy Sci. 57:1504-1508.

Bottazzi, V., B. Battistotti, and F. Bianchi. 1982. The microscopic crystalline inclusions in Grana cheese and their x-ray microanalysis. Milchwissenschaft 37:577-580.

Bottazzi, V., F. Lucchini, A. Rebecchi, and G. L. Scolari. 1994. I cristalli del formaggio grana [Crystals present in Grana cheese]. Sci. Tecn. Latt. Cas. 45:7-14. (In Italian).

Cattaneo, S., J. A. Hogenboom, F. Masotti, V. Rosi, L. Pellegrino, and P. Resmini. 2008. Grated Grana Padano cheese: new hints on how to control quality and recognize imitations. Dairy Sci. Technol. 88:595-605.
Codex Alimentarius. 1978. Codex General Standard for Cheese: Standard 283-1978. World Health Organization. Accessed Jan. 12, 2016. http://www.fao.org/fao-who-codexalimentarius/standards/ en/.

Cremonesi, P., G. Perez, G. Pisoni, P. Moroni, S. Morandi, M. Luzzana, M. Brasca, and B. Castiglioni. 2007. Detection of enterotoxigenic Staphylococcus aureus isolates in raw milk cheese. Lett. Appl. Microbiol. 45:586-591.

Culka, A., J. Jehlička, and H. G. M. Edwards. 2010. Acquisition of Raman spectra of amino acids using portable instruments: Outdoor measurements and comparison. Spectrochim. Acta A Mol. Biomol. Spectrosc. 77:978-983.

D'Incecco, P., F. Faoro, T. Silvetti, K. Schrader, and L. Pellegrino. 2015. Mechanisms of Clostridium tyrobutyricum removal through natural creaming of milk: A microscopy study. J. Dairy Sci. 98:5164-5172.

Dalas, E., P. Malkaj, Z. Vasileiou, and D. G. Kanellopoulou. 2008. The effect of leucine on the crystal growth of calcium phosphate. J. Mater. Sci. Mater. Med. 19:277-282.

Gatti, M., B. Bottari, C. Lazzi, E. Neviani, and G. Mucchetti. 2014. Invited review: Microbial evolution in raw-milk, long-ripened cheeses produced using undefined natural whey starters. J. Dairy Sci. 97:573-591.

Gaucheron, F., Y. Le Graët, F. Michel, V. Briard, and M. Piot. 1999. Evolution of various salt concentrations in the moisture and in the outer layer and centre of a model cheese during its brining and storage in ammoniacal atmosphere. Lait 79:553-566.

Giolitti, G., and G. F. Mascherpa. 1970. La formazione di depositi di tirosina in formaggi a lunga stagionatura. L'Industria del Latte 6:83-85.

Grosse Daldrup, J. B., C. Held, F. Ruether, G. Schembecker, and G. Sadowski. 2009. Measurement and modeling solubility of aqueous multisolute amino-acid solutions. Ind. Eng. Chem. 49:1395-1401.

Heertje, I., M. J. Boskamp, F. Van Kleef, and F. H. Gortemaker. 1981. The microstructure of processed cheese. Neth. Milk Dairy J. 35:177-179.

ISO (International Organization for Standardization). 2004a. ISO Standard No. 1735: Cheese and processed cheese products-Determination of fat content - Gravimetric method. ISO, Geneva, Switzerland.

ISO (International Organization for Standardization). 2004b. ISO Standard No. 5534: Cheese and processed cheese-Determination of the total solid content (reference method). ISO, Geneva, Switzerland.

ISO (International Organization for Standardization). 2005. ISO Standard No. 13903: Animal feeding stuffs - Determination of amino acids content (reference method). ISO, Geneva, Switzerland.

ISO (International Organization for Standardization). 2008. ISO Standard No. 5545: Rennet caseins and caseinates-Determination of ash (reference method). ISO, Geneva, Switzerland.

ISO (International Organization for Standardization). 2011. ISO Standard No. 27871: Cheese and processed cheese-Determination of the nitrogenous fractions. ISO, Geneva, Switzerland.

Jeanson, S., J. Floury, V. Gagnaire, S. Lortal, and A. Thierry. 2015. Bacterial colonies in solid media and foods: A review on their growth and interactions with the micro-environment. Front. Microbiol. 6:1284.

Jenkins, A. L., R. A. Larsen, and T. B. Williams. 2005. Characterization of amino acids using Raman spectroscopy. Spectrochim. Acta A Mol. Biomol. Spectrosc. 61:1585-1594.

Kaláb, M., J. Yun, and S. H. Yiu. 1987. Textural properties and microstructure of process cheese food rework. Food Microbiol. 6:181-192.

Koutsopoulos, S. 2002. Synthesis and characterization of hydroxyapatite crystals: A review study on the analytical methods. J. Biomed. Mater. Res. 62:600-612.

Le Boucher, C., V. Gagnaire, V. Briard-Bion, J. Jardin, M. B. Maillard, G. Dervilly-Pinel, B. Le Bizec, S. Lortal, S. Jeanson, and A. Thierry. 2016. Spatial distribution of Lactococcus lactis colonies modulates the production of major metabolites during the ripening of a model cheese. Appl. Environ. Microbiol. 82:202-210. 
Marcos, A. 1993. Water activity in cheese in relation to composition, stability and safety. Pages 439-469 in Cheese: Chemistry, Physics and Microbiology, Vol. 1. P. F. Fox, ed. Chapman \& Hall, London, UK.

Masotti, F., J. A. Hogenboom, V. Rosi, I. De Noni, and L. Pellegrino. 2010. Proteolysis indices related to cheese ripening and typicalness in PDO Grana Padano cheese. Int. Dairy J. 20:352-359.

McPherson, A., S. B. Larson, and Y. G. Kuznetsov. 2012. Tyrosine microcrystals produced by digestion of proteins with pancreatic enzymes. Cryst. Growth Des. 12:3594-3602.

McSweeney, P. L. H. 2004. Biochemistry of cheese ripening. Int. J. Dairy Technol. 57:127-144.

Ong, L., R. R. Dagastine, S. E. Kentish, and S. L. Gras. 2010. Transmission electron microscopy imaging of the microstructure of milk in cheddar cheese production under different processing conditions. J. Food Sci. 75:135-145.

Pellegrino, L., G. Battelli, P. Resmini, P. Ferranti, F. Barone, and F. Addeo. 1997. Effects of heat load gradient occurring in moulding on characterization and ripening of Grana Padano. Lait 77:217228 .

Sauer, G. R., W. B. Zunic, J. R. Durig, and R. E. Wuthier. 1994 Fourier transform Raman spectroscopy of synthetic and biological calcium phosphates. Calcif. Tissue Int. 54:414-420.

Schrader, K. 2012. Structural analysis of milk and milk products. Deutsche Molkerei-Zeitung 133:30-34.
Shock, A. A., W. J. Harper, A. M. Swanson, and H. H. Sommer. 1948 What's in those "white specks" on Cheddar. Wisc. Agric. Exp. Stn. Bull. 474:31-32.

Silva, J. V. C., P. D. S. Peixoto, S. Lortal, and J. Floury. 2013. Transport phenomena in a model cheese: The influence of the charge and shape of solutes on diffusion. J. Dairy Sci. 96:6186-6198.

Tansman, G. F., P. S. Kindstedt, and J. M. Hughes. 2014. Powder x-ray diffraction can differentiate between enantiomeric variants of calcium lactate pentahydrate crystal in cheese. J. Dairy Sci. 97:7354-7362.

Tansman, G. F., P. S. Kindstedt, and J. M. Hughes. 2015. Crystal fingerprinting: Elucidating the crystals of Cheddar, ParmigianoReggiano, Gouda, and soft washed-rind cheeses using powder x-ray diffractometry. Dairy Sci. Technol. 95:651-664.

Tlili, M. M., M. Ben Amor, C. Gabrielli, S. Joiret, G. Maurin, and P. Rousseau. 2001. Characterization of $\mathrm{CaCO}_{3}$ hydrates by microRaman spectroscopy. J. Raman Spectrosc. 33:10-16.

Tuckey, S. L., H. A. Ruehe, and G. L. Clark. 1938. X-ray diffraction analysis of white specks in Cheddar cheese. J. Dairy Sci. 21:161.

Washam, C. J., T. J. Kerr, V. J. Hurst, and W. E. Rigsby. 1985. A scanning electron microscopy study of crystalline structures on commercial cheese. Dev. Ind. Microbiol. 26:749-761. 